\title{
Percepciones en torno al exceso de peso en el contexto sociocultural
}

Maribel Candelaria Martínez'

Centro Universitario del Sur -

Universidad de Guadalajara

\author{
Edgardo Leija Irurzo ${ }^{2}$ \\ El Colegio de México
}

\section{Ensayo}

Material original autorizado para su primera publicación en Journal de Ciencias Sociales, Revista Académica de la Facultad de Ciencias Sociales de la Universidad de Palermo.

Recepción: 25-11-2020

Aceptación: 10-03-2021

Resumen: El exceso de peso es un padecimiento que actualmente es responsable del aumento en los riesgos de padecer diabetes, hipertensión y algunos tipos de cáncer. Este fenómeno social requiere de un tratamiento multidisciplinario en el que estén involucrados estudiosos de la salud y del comportamiento humano, desde personal de medicina, nutrición, activación física, trabajo social y psicología; hasta sociólogos, antropólogos, historiadores y otros científicos sociales que contribuyan a comprender mejor la impronta de esta problemática en contextos específicos. Aunque existan diferencias entre estas disciplinas en la forma de abordar y percibir dicho fenómeno, estas no deben de anularse, sino complementarse entre sí. Al considerar las distintas perspectivas y enfoques de estas disciplinas en torno al exceso de peso se podrá visualizar esta problemática en su totalidad, es decir, las pautas y circunstancias bajo las cuales se desarrolla. Por tanto, el objetivo de la presente investigación es examinar la ambivalencia y mutabilidad de la percepción en torno al exceso de peso. La percepción que se ha dado al exceso de peso ha cambiado a lo largo de la historia, de concebirse como algo normal, e incluso positivo, pasó a tener una connotación negativa. Esta modificación de la percepción se ha visto reflejada en la sociedad contemporánea, y el impacto que actualmente tiene se ve reflejado en el aumento constante

\footnotetext{
1 Licenciada y Magister en Psicología. Estudiante del Doctorado en Psicología con Orientación en Calidad de Vida y Salud.

Correo electrónico: mcandelariamtz@gmail.com

${ }^{2}$ Licenciado y Magister en Historia. Estudiante de Doctorado en Historia en el Centro de Estudios Históricos.

Correo electrónico: eleija@colmex.mx
} 
de la población con exceso de peso. La ambivalencia genera déficit en el abordaje y las estrategias carecen de unificación en los distintos sectores, se vislumbra un escenario complejo ante las distintas posturas y la falta de un abordaje transdisciplinario. Se requiere cambios en la concepción del fenómeno alejados de la culpa y asumiendo responsabilidades tanto a nivel individual como colectivo.

Palabras clave: exceso de peso; percepción; estética; estigmatización.

\section{Perceptions about excess weight in the sociocultural context}

Abstract: Excess weight is a condition that is currently responsible for the increased risks of diabetes, hypertension and some types of cancer. This social phenomenon requires a multidisciplinary treatment in which students of health and human behavior are involved, from medicine, nutrition, physical activation, social work and psychology personnel; to sociologists, anthropologists, historians and other social scientists who contribute to better understand the imprint of this problem in specific contexts. Although there are differences between these disciplines in the way of approaching and perceiving this phenomenon, they should not cancel each other out, but rather complement each other. By considering the different perspectives and approaches of these disciplines around excess weight, this problem can be visualized in its entirety, that is, the guidelines and circumstances under which it develops. Therefore, the objective of the present research is to examine the ambivalence and mutability of the perception around excess weight. The perception that has been given to excess weight has changed throughout history, from being conceived as something normal, and even positive, to have a negative connotation. This change in perception has been reflected in contemporary society, and the impact it currently has is reflected in the constant increase in the overweight population. Ambivalence generates a deficit in the approach and the strategies lack unification in the different sectors, a complex scenario is envisioned in the face of the different positions and the lack of a transdisciplinary approach. Changes are required in the conception of the phenomenon away from guilt and assuming responsibilities both individually and collectively.

Keywords: overweight; perception; aesthetics; stigmatization.

\section{Introducción}

Este trabajo aborda la percepción que se tiene sobre el exceso de peso, para ello se apoya en algunas nociones históricas en torno al cuerpo y la belleza. Toma en cuenta algunos parámetros socioculturales que han influido en su concepción, la forma como se ha desarrollado y transformado con el paso del tiempo hasta alcanzar una connotación dentro 
de la salud y el bienestar, pero también como descuido e irresponsabilidad. Objetivo: examinar la ambivalencia y mutabilidad de la percepción en torno al exceso de peso.

El exceso de peso involucra distintos aspectos: genéticos, ambientales, sociales y del estilo de vida (Rivera Dommarco et al. 2018). Por tanto, requiere la suma de esfuerzos de diversos sectores sociales: familiares, profesionales y políticos. Si bien se ha trabajado desde diferentes ámbitos, ha resaltado de manera notable el abordaje desde el enfoque biomédico sobre el enfoque biopsicosocial. Hoy en día, una diferencia que se puede observar en la sociedad es el reconocimiento que existe sobre el exceso de peso como problema de salud, en ello han influido las distintas disciplinas. Sin embargo, sólo se reconoce la problemática y difícilmente la solución.

Mientras el modelo biomédico se refiere al exceso de peso desde una concepción patológica, perspectivas más recientes -entre ellas las de las ciencias sociales- utilizan el término gordura para contrastar la concepción patológica que puede presentarse desde el momento en que se identifica como una enfermedad (Energici y Acosta, 2020). Dicha discrepancia entre las disciplinas y sus enfoques se proyecta en ciertos sectores de la sociedad ya que se tiene la idea de que el exceso de peso es una manifestación de bienestar, belleza y buena salud (Marcos et al. 2010), mientras que en otros sectores más favorecidos económicamente se visualiza como enfermedad. En este sentido, se puede observar que la forma de acercarse al fenómeno va a determinar la interacción y por tanto las acciones a seguir. Además, el contexto y las circunstancias van a ser dos variables imprescindibles para analizarlo, lo que implica profundizar en su origen y desenvolvimiento para transformarlo y erradicarlo.

Antes de continuar es imprescindible señalar que el exceso de peso, como problema de índole social, puede ser abordado teóricamente desde diferentes enfoques y escuelas de pensamiento, particularmente si se busca comprender su percepción. Para la sociología de Max Weber, las creencias, sentimientos y sistema de valores traspasados generacionalmente no solamente coexisten y se reproducen en las sociedades, sino que también pueden ser capaces de transformarse. En este sentido, la forma de percibir y representar el mundo va a ser fundamental para distinguir a las sociedades y sus miembros, pero también será dominante en el pensamiento humano y en el cambio histórico al que se enfrentan (Macionis y Plummer, 2011).

Para Emile Durkheim las estructuras preestablecidas de la sociedad, o hechos sociales cuya realidad se extiende más allá de la experiencia y percepción de los individuos, son articuladas por pautas de la conducta humana. Por ello, la sociedad se vuelve determinante de los pensamientos y acciones de los individuos, los cuales no pueden ser estudiados de manera aislada ya que nunca se capturaría la esencia humana dentro del 
organismo complejo llamado sociedad, ni mucho menos el principio de su función (Macionis y Plummer, 2011).

Otro autor que suma al cometido de este ensayo es Serge Moscovici y su concepto de la representación social, un cúmulo de conocimientos organizados que tienen la función de amalgamar la comunicación y el comportamiento de los individuos; una actividad psíquica con la cual se hace inteligible la realidad. Dicha representación se encuentra integrada en los diferentes grupos sociales y en la cotidiana interacción de sus intercambios (Mora, 2002).

Pero, ¿es el exceso de peso un fenómeno reciente? ¿Cuáles de los elementos teóricos antes mencionados podemos encontrar en su análisis? ¿Cuáles han sido algunos de los parámetros que han marcado la percepción respecto a este padecimiento? ¿Qué elementos culturales o mediáticos contribuyen a su connotación? ¿Por qué existe un estigma respecto a esta enfermedad? ¿Qué papel juegan los expertos de la salud y científicos sociales en el tema? Sirvan las siguientes páginas para buscar responder dichas interrogantes.

\section{Representaciones corpóreas, de lo ordinario a lo inusual}

Durante el devenir de la humanidad, el exceso de peso ha sido percibido de distintas formas de acuerdo al contexto en donde se refleja y a la sociedad que lo concibe, es decir, se ha vislumbrado como parte de una representación social de la realidad que incorpora los comportamientos e interacciones en torno a dicho fenómeno. De esta manera, el exceso de peso ha sido comprendido, bajo ciertos parámetros, tanto por el grupo ostentador, como por el grupo desposeído de esta singularidad.

Al analizar retrospectivamente la Boda de campesinos (1568) de Pieter Bruegel el Viejo, quien plasmó las actividades habituales de una población rural europea y centró su humanismo en la aceptación pasiva de su destino (Charles, 2007), se pueden identificar ciertos elementos que, directa y colateralmente, advierten la existencia del exceso de peso en estas sociedades en dónde los recursos escaseaban y su alimentación se supeditaba a lo cosechado y criado, incluso en eventos festivos.

En dicha obra pictórica, la escena principal es una celebración cuya socialización, entendida como aquella experiencia que dota al individuo de normas de interacción y asimilación de pautas culturales (Macionis y Plummer, 2011), es un banquete en el cual el intercambio y la comunicación son una constante de esta representación social. En torno a la mesa principal se encuentran los comensales que platican y degustan los alimentos, también discurren los músicos, los invitados que arriban al evento y los encargados de servir la comida; uno de ellos sirve la bebida y otros más aproximan los platos a la mesa para ser 
distribuidos por una persona que ocupa un asiento en la mesa. Mientras esto acontece, una niña sentada en el suelo parece saborear su platillo llevándose el dedo a la boca. Si bien es cierto que los adultos prevalecen en la representación, los niños no son excluidos totalmente de esta, pues al igual que la niña antes mencionada, otros dos infantes forman parte de los comensales; además, parecen reproducir los mismos hábitos y conductas alimentarias de sus progenitores.

De cierta forma, el comportamiento y las conductas de los individuos se supeditan a la estructura social, de ahí que lo percibido por ellos se vuelve una representación del mundo y de la experiencia adquirida en esa realidad. El individuo se reconoce así mismo, en tanto la sociedad lo construya a él bajo su sistema de creencias y valores, cualidades que llegan a ser heredadas o transfiguradas con el paso de los años.

Volviendo a la escena de Bruegel, la gruesa corporalidad de los involucrados en el convite parece ser un común denominador que, de manera inadvertida, forma parte del contexto y de las circunstancias de la época, es decir, de su cotidianidad. Conscientes del anacronismo que conlleva plasmar parámetros actuales en sucesos del pasado, bien podría pensarse que el exceso de peso siempre ha estado latente a lo largo de la historia, aunque sin la misma connotación negativa, estigma o prejuicio al que se le somete hoy en día. En este sentido, es difícil asegurar que el exceso de peso es un fenómeno reciente.

Para los antiguos griegos la belleza del cuerpo humano se exteriorizaba a través de las partes del cuerpo humano, las cuales eran selectivas según su valor cosmético (desde el rostro hasta los pies), y por medio de su motricidad y gesticulación (movimientos de la rodilla, brazos, sonrisa, labios, lenguaje, etc.). La belleza era un atributo natural que pertenecía al reino de los sentidos y ejercía su influencia seductora y cierto carácter moral sobre su ostentador. Un hombre hermoso por lo general era valiente y fuerte, en tanto que una mujer asimétrica se valoraba como desleal. Para Aristóteles, la simetría era una de las tres categorías matemáticas que constituían a la belleza, por lo que dos cuerpos simétricos (hombre-mujer) debían de ser conmensurables bajo el mismo patrón (Bayer, 2012).

Desde el renacimiento, el cuerpo humano debía de poseer ciertas proporciones en su representación, pues tenía que ser percibido de manera armoniosa con el objeto de generar en el ojo humano el mismo efecto que una nota musical bien ejecutada producía en el oído. Según da Vinci cada parte del todo debía de ajustarse a su unidad y este principio era aplicable al cuerpo humano (da Vinci, 1989). En este sentido, la anchura humana debía de comprender la misma distancia que la existente entre la parte superior de sus caderas y la inferior de sus glúteos (estando el cuerpo de pie sobre sus dos piernas) y la desplegada entre la parte superior de la cabeza hasta su axila. Por encima de las caderas, la cintura tendría que estar a medio camino de la distancia existente entre las axilas y la parte inferior de sus glúteos (da Vinci, 1989). 
Estos cánones corpóreos también se manifestaron en otra de las artes plásticas por excelencia, la escultura. Para el siglo XVIII, los límites de esta forma de representación humana llegaron a percibirse como aquellos que "consisten en atenerse lo más cercanamente posible a las formas más perfectas del cuerpo humano" (Bayer, 2012, p. 229).

Cabe destacar que dicha geometría corporal fue un elemento ideal, y en la mayoría de los casos y representaciones este se desvaneció, especialmente cuando se pretendió reflejar más la naturaleza humana y su realidad. No obstante, nunca se abandonó por completo dicho idealismo, pues este influiría decisivamente en la percepción de belleza y fealdad corporal. El empirista ilustrado inglés David Hume señaló que, como parte de nosotros, el cuerpo podía desprender alguna de estas dos relaciones estéticas y éstas a su vez podían ser causantes de orgullo y humildad, respectivamente. En palabras del propio Hume (2012):

La belleza, del género que sea, nos proporciona un propio deleite o satisfacción, y la fealdad produce dolor. Si la belleza o la fealdad pertenecen a nuestro propio cuerpo, este placer o dolor se convierten en orgullo o humildad [...] Placer y dolor [...] acompañantes necesarios de la belleza y la fealdad [...] constituyen su verdadera esencia [sin embargo] no es sólo la belleza del cuerpo lo que produce orgullo, sino también su vigor y fuerza. (p. 257-259)

Bajo estos parámetros, el cuerpo humano podía percibirse con admiración o aversión, aunque el exceso de peso continuó siendo interpretada de manera dicotómica, es decir, ocasionalmente representó abundancia, felicidad y salud, mientras que, por otro lado, se fue distinguiendo cada vez más como un mal menor. Así lo demuestra la publicidad comercial de finales del siglo XIX y principios del XX, específicamente la que comenzó a patrocinar a la incipiente industria farmacéutica, tanto la de medicamentos de patente como la de aquellos confeccionados aún en menor escala.

En su tarea de nutrir y fortificar, la famosa Emulsión Scott llegó a enunciar en 1904 portentosas transformaciones de niños débiles y endebles (esbeltos) a niños robustos, colorados y atléticos (obesos); así lo demuestra algunos de sus elementos visuales utilizados de manera propagandística (Muro, 1904, p. 4). De manera adyacente, el exceso de peso fue denotado paulatinamente como un padecimiento de la salud, prueba de ello fueron los anuncios que cuestionaban al lector sobre su nivel de exceso de peso, sugiriéndoles tratamientos con píldoras reductoras, garantizadas con la disminución semanal del peso y la nulidad de secuelas en su organismo (Agüeros, 1894, p. 2).

Poco a poco la publicidad jugó un papel clave en la aceptación y en el inconformismo corporal. Asimismo, dio cabida a un gran número de productos milagrosos que prometieron curar el exceso de peso en nombre de la ciencia médica, pero que de manera ilusoria sólo se ampararon en los avances farmacéuticos de la época, los cuales se ensancharían entrado el siglo XX. 


\section{Estigma y re-significación social de los agentes}

Actualmente, conceptualizar el exceso de peso como una diferencia entre la ingesta de calorías y el gasto de las mismas al momento de plantear programas reduce las variables a intervenir y por lo tanto el éxito. Es necesario contemplar el contexto en el que se desarrolla e identificar que originó el padecimiento, pues esto hace la diferencia entre considerarlo como acciones independientes al comportamiento o consecuencia del mismo (Matus et al. 2015), es decir, retomar las percepciones que se tienen sobre el exceso de peso y la figura corporal. En su mayoría los trabajos realizados sobre el exceso de peso se han centrado en abordar la problemática desde la prevención y el tratamiento, se retoma a la persona como un agente pasivo/a que necesita ser modificado, por otro lado, se presenta al profesional de la salud como el experto y conocedor que deposita todos sus conocimientos a la causa, sin tomar en cuenta las verdaderas necesidades de la persona: sus percepciones, inquietudes, pensamientos, emociones y estilos de vida, consideraciones que promueven la revaloración de la persona en su conjunto, es decir, ir más allá de posicionarla solamente como un ente pasivo.

Una forma de dar complementariedad al tratamiento y retomar a la persona como agente activo es incorporar la perspectiva social, al intervenir principalmente los actores como protagonistas y no como aquellos a los que se tenga que modificar, lo cual se aborda sustancialmente con la teoría de las representaciones sociales (Quintero et al. 2016). De tal manera, se deben de contemplar más variables intervinientes para comprender mejor la realidad de la persona con exceso de peso, cuyo comportamiento se deriva de su interacción con el entorno y del constante flujo de intercambios que esta tiene con él.

Los factores socioculturales que intervienen son diversos, por ejemplo, el ámbito familiar, el ambiente obesogénico y el contexto sociocultural; además, la imagen corporal que constantemente se ve influenciada por la publicidad y los cánones estéticos. Los estereotipos de belleza impuestos por la sociedad han llevado a las mujeres a inmiscuirse frecuentemente en tratamientos para disminuir su peso y por lo tanto obtener una figura aceptable (Barragán et al. 2018). Lo que genera costos sociales altos tanto en hombres como en mujeres debido a la discriminación ocasionada por estereotipos y prejuicios (Pearl et al. 2018). Las personas con exceso de peso tienen desventaja en instituciones educativas (Golaszewski et al. 2018), en los servicios de salud (Barra y Singh, 2018) y en actividades de recreación tales como hoteles y restaurantes (Poria, et al. 2021); asimismo, perciben salarios más bajos y presentan ausentismo laboral según la Organización para la Cooperación y Desarrollo Económico (Agencia Reforma, 20 de mayo de 2017). Comúnmente, estos estereotipos de belleza suelen 
ser "imágenes distorsionadas y cargadas de prejuicios acerca de toda una categoría de personas" (Macionis, 2011, p. 302), incluidas las que padecen exceso de peso.

A nivel global, se ha llegado al punto de comercializar la salud debido a la acelerada vorágine de consumo en la que se han insertado distintas sociedades (urbanas, rurales, centrales, periféricas, desarrolladas o en vías de desarrollo). Aprovechándose de esto, las grandes empresas, principalmente las que se dedican a la fabricación y venta de bienes de consumo (productos farmacéuticos, cosméticos, bebidas y alimentos, etc.), prometen desaparecer aquella alteración que no coincide con los actuales estándares de belleza. Es en este contexto en el cual la salud se mercantiliza, pues múltiples productos son adquiridos para bajar de peso con la finalidad de obtener un cuerpo delgado. Estos productos se promocionan diariamente para adquirir una imagen aceptada por la sociedad, una imagen que la mercadotecnia ha construido, lo que lleva a pensar que existe gran cantidad de dinero que se mueve a alrededor de la salud o de lo que se pretende negociar bajo este término, ya que algunas empresas sólo la ven como una forma de generar riqueza.

En la sociedad actual, la persona con exceso de peso se visualiza como irresponsable, perezosa y con falta de fuerza de voluntad (Randall et al. 2017), tal y como se menciona en Wharton et al. (2020, p. 876): "la narrativa cultural dominante sobre la obesidad alimenta las suposiciones sobre la irresponsabilidad personal y la fuerza de voluntad y echa la culpa y la vergüenza a las personas que viven con obesidad"

En este sentido, el exceso de peso visto como enfermedad, tal como lo propone el modelo biomédico y las grandes empresas beneficiadas por el consumo que se genera con la denominada epidemia del exceso de peso, tiene consecuencias sociales como estigmatización y discriminación y crea percepciones que pueden llevar al no reconocimiento del tamaño del cuerpo por no querer aceptar las características negativas que se asocian a las personas con exceso de peso (Rivera Dommarco et al. 2012). El estigma también conlleva desajuste psicológico conformado desde edades tempranas en el núcleo familiar, donde pueden aparecer patrones de conducta expuestas por los cuidadores o padres. La estigmatización de este padecimiento no solo trae consecuencias para los afectados, sino para la sociedad en general, incluida la educación y el compromiso que deben de poseer todas aquellas profesiones que procuran la salud y el bienestar, pues sus expertos no están exentos de discriminar a las personas con exceso de peso. Gómez-Pérez et al. (2017) describen cómo el personal de salud refleja cotidianamente actitudes discriminatorias en sus prácticas, ya que suelen omitir recomendaciones en el tratamiento, dedicar menos tiempo en la consulta y responsabilizar a la persona por su condición.

Deslindarse de la responsabilidad, más allá de afrontar el problema, limita la solución. Si bien existen esfuerzos que pretenden regular los establecimientos que distribuyen 
alimentos con alto contenido calórico e influir con ello en el combate de esta epidemia -como el Acuerdo Nacional para Salud Alimentaria. Estrategia contra el Sobrepeso y la Obesidad(Rodríguez-Torres y Casas-Patiño, 2018), difícilmente su implementación se lleva a cabo por la falta de coordinación y compromiso entre los distintos sectores sociales (públicos y privados). Aunado a lo anterior, la responsabilidad de padecer exceso de peso recae completa y únicamente en la persona, cuya decisión, en la forma de alimentarse y ejercitarse, corresponde a ella. Sin embargo, la elección y el acceso a los alimentos y a espacios de acondicionamiento físico dependen de otras determinantes, tanto socioeconómicas como culturales. En este sentido, los representantes públicos tienen la responsabilidad de informar al individuo sobre las consecuencias y beneficios de adoptar ciertos hábitos y crear espacios seguros y adecuados para realizar actividad física. Tanto el sector público como el privado podrían fomentar el desarrollo de actividad física, así como desarrollar comedores propicios para favorecer los hábitos saludables.

Al ser el exceso de peso un problema de salud mundial -particularmente de países en vías de desarrollo- los esfuerzos para minar su impacto en la sociedad deberían de ser compartidos. Desde políticas públicas encaminadas a procurar su prevención e inhibición con campañas de salud más efectivas y cercanas a la población, hasta mecanismos regulatorios más estrictos en los productos manufacturados y comercializados que directa e indirectamente inciden en su propagación y erróneo tratamiento. A ello se debe de sumar la labor académica, en todas sus dimensiones científicas, y el compromiso de la iniciativa privada con responsabilidad social.

\section{Conclusiones}

A través de los años se ha transformado la forma de vislumbrar el exceso de peso, al grado de conceptualizarlo primero como algo común y después como algo negativo y despreciable, es decir, aquello a lo que no se debe aspirar. Frecuentemente se convive con esta última noción, la cual se arraiga en las personas desde su infancia hasta considerarla una verdad absoluta en su fase adulta, es decir, sin cuestionar dicho paradigma o identificar el contexto y las circunstancias de quienes padecen y viven esta enfermedad. De ahí la necesidad de generar oportunidades de conocimiento de otras concepciones y de modificar no sólo la validez de ciertos cánones y perspectivas, sino de acciones en torno a las personas con exceso de peso. Con este fundamento se puede asumir un discurso tolerante y conciliador que no desestime la opinión del otro por ser distinta a la concebida.

Al ser el exceso de peso un fenómeno social que ha estado presente históricamente, su identificación no se restringe solamente a un problema de salud reciente. La mutabilidad de su interpretación, supeditada a paradigmas de distinta índole (sociales, culturales, 
económicos, psicológicos, etc.), ha complejizado el consenso en torno a su existencia, lo que evidencia la ambivalencia de su percepción.

Si no existe disposición para cambiar la forma de ver el fenómeno la tarea puede llegar a ser ardua y sinuosa para todos aquellos involucrados en el proceso. Además, si a ello se le añade la obtención de beneficios económicos la labor se complica aún más, y el sesgo tanto en investigación como en la atención clínica se convierte en una limitación para descubrir y plantear nuevas estrategias que favorezcan a la sociedad al momento de buscar opciones de tratamiento.

Una de las posibles razones del fracaso en el tratamiento del exceso de peso es que los principales determinantes del cambio no se deben enfocar exclusivamente en la alimentación y la actividad física a través de campañas educacionales, sino en la modificación del ambiente en todas sus dimensiones, especialmente en los elementos culturales y mediáticos que contribuyen a su connotación. En un entorno social desfavorable, la publicidad, costos y accesibilidad de bebidas azucaradas y alimentos grasos tienen mayores niveles de consumo que aquellos productos menos disponibles en el mercado o que son accesibles a él encarecidamente (por ejemplo, ciertas frutas, verduras, y alimentos saludables). La educación escolar, desde la infancia, es un claro ejemplo de un entorno desfavorable, y la facilidad de utilizar las máquinas expendedoras de bebidas con alto contenido de azúcar inhibe toda estrategia contra el exceso de peso y potencializa su presencia.

Si bien es cierto que, desde una perspectiva sociocultural, hay elementos teóricos capaces de sumar al análisis del exceso de peso (estructura y representación social, percepciones y experiencias, comportamientos e intercambios culturales), también se vuelve fundamental comprender los parámetros que han marcado la percepción y estigmatización de este padecimiento (estéticos, publicitarios, etc.).

Un fenómeno complejo como el exceso de peso requiere consenso entre la comunidad científica y los distintos profesionistas involucrados en su abordaje, pues es necesario que se contemplen todas las variables para obtener resultados positivos, por tanto, se requiere incluir constantemente nuevos conocimientos desde diferentes ámbitos, o bien deconstruir el conocimiento para crear nuevas formas de interacción. En la medida en que los profesionistas de la salud, apoyados en los científicos sociales, analicen sus propias creencias y modos de proceder ante las personas con exceso de peso, se contribuirá a transformar el estigma imperante respecto a esta enfermedad y, por ende, se podrá disminuir su discriminación en el ambiente sanitario. Por tal motivo, el papel que juegan los expertos sociales y de la salud es trascendental, especialmente cuando se trata de una problemática cuya erradicación puede contribuir a mejorar la calidad de vida humana. 
Los autores agradecen la beca de manutención para estudios de doctorado otorgada por el Consejo Nacional de Ciencia y Tecnología (CONACyT).

\section{Referencias bibliográficas}

Barra, M. y Singh, S., (2018). Too big to be seen: weight-based discrimination among nursing students. Nursing Forum 53(4), 529-534. doi: 10.1111/nuf.12282

Barragán, R., Rubio, L., Portolés, O., Asensio, E., Ortega, C., Sorli, J. y Corella, D. (2018). Estudios de investigación cualitativa sobre las diferencias entre los hombres y mujeres en la percepción de la obesidad, sus causas, abordaje y repercusiones para la salud. Nutrición Hospitalaria, 35(5), 1090-1099. doi: 10.20960/nh.1809

Bayer, R. (2012). Historia de la estética. Fondo de Cultura Económica.

Charles, V. (2007). El Renacimiento. Numen.

Energici, M., y Acosta, E. (2020). El estudio de la obesidad y la gordura desde la Sociología y la Psicología Social. Athenea Digital, 20(2), 1-19.

Golaszewski, N., Pasch, K., Fernandez, A., Poulos, N., Batanova, M. y Loukas, A. (2018). Perceived weight discrimination and school connectedness among youth: Does teacher support play a protective role? Journal School Health, 88(10), 754-761. doi: 10.1111/josh.12682

Gómez-Pérez, D., Ortiz, M. y Saiz, J. (2017). Estigma de obesidad, su impacto en las víctimas y en los equipos de salud: una revisión de la literatura. Revista Médica de Chile, 145, 1160-1164.

Hume, D. (2012). Tratado de la naturaleza humana. Ensayo para introducir el método del razonamiento humano en los asuntos morales. Porrúa.

Macionis, J. y Plummer, K. (2011). Sociología. Pearson.

Marcos, L. Rodríguez, L. Pérez, M. y Caballero, M. (2010). Trastornos de la conducta alimentaria. Trastornos de la Conducta Alimentaria, 11, 1231-1258.

Matus, N., Álvarez, G., Nazar, D. y Mondragón, R. (2015). Percepción de adultos con sobrepeso y obesidad y su influencia en el control de peso en San Cristóbal de las Casas, Chiapas. Estudios Sociales, 47, 381-409. 
Mora, M. (2002). La teoría de las representaciones sociales de Serge Moscovici. Athenea Digital, 2, 1-25.

Pearl, R., Wadden, T., Tronieri, J., Chao, A., Alamuddin, N. y Berkowitz, R.I, (2018). Everyday discrimination in a racially diverse sample of patients with obesity. Clinical Obesity 8(2), 140-146. doi: 10.1111/cob.12235

Poria, Y., Beal, J. y Shani, A. (2021). "I am so ashamed of my body": Obese guests' experiences in hotels. International Journal of Hospitality Management, 92. doi: 10.1016/j.jhm.2020.102728

Quintero, Y., Villarroel. J., Pargas, L., Bastardo, G., Angarita, C., Rivas, J. y Castañeda, G. (2016). La Teoría de las representaciones Sociales, su aplicación en los estudios de salud y enfermedad: el caso de la obesidad. Revista Facultad de ciencias de la Salud UDES, 138-148.

Randall, J., Zimmer, C., O'Brien, K., Trump-Steele, R., Villado, A.J. y Hebl, M, (2017). Weight discrimination in helping behavior. Revue Européenne de Psychologie Appliqueée/Eur, 6(3), 125-137.doi: 10.1016/j.erap.2017.02.002

Rivera Dommarco, J. A., Colchero, M. A., Fuentes, M. L., González de Cosío, T., Aguilar Salinas, C., Hernández Licona, G., Barquera, S., García Chávez, C. G., Unar Mungía, M. y Hernández Fernández, M. (2018). Postura. Recomendaciones para una política de Estado para la prevención y control de la obesidad en México en el periodo 2018-2024. En J.A. Rivera Dommarco, M. Colchero, M. L. Fuentes, T. González de Cosío, C. Aguilar Salinas, G. Hernández Licona y S. Barquera (Eds.), La obesidad en México. Estado de la política pública y recomendaciones para su prevención y control (pp.15-30). Instituto Nacional de Salud Pública.

Rivera Dommarco, J. A., Velasco Bernal, A., Hernández Ávila, M., Aguilar Salinas, C. A., Vadillo Ortega, F. y Murayama Rendón, C. (2012). Trabajo de postura. Obesidad en México: recomendaciones para una política de Estado. En J. A. Rivera Dommarco, M. Hernández Ávila, C. A. Aguilar Salinas, F. Vadillo Ortega y C. Murayama Rendón (Eds.), Obesidad en México: recomendaciones para una política de Estado (pp. 6-45). Instituto Nacional de Salud Pública.

Rodríguez-Torres, A. y Casas-Patiño, D. (2018). Determinantes sociales de la obesidad en México. Revista de Enfermería del Instituto Mexicano del Seguro Social, 26(4), 281-290.

Vinci da, L. (1989). Cuaderno de notas. Industrias Gráficas MARAL. 
Wharton, S., Lau, D., Vallis, M., Sharma, A., Biertho, L., Campbell-Scherer, D., Adamo, K., Alberga, A., Bell, R., Boulé, N., Boyling, E., Brown, J., Calam, B., Clarke, C., Crowshoe, L., Divalentino, D., Forhan, M., Freedhoff, Y., Gagner, M., Glazer, S., Grand, C. ....(2020). Obesity in adults: a clinical practice guideline. Canadian Medical Association Journal, 192(31), 875-891. doi: 10.1503/cmaj.191707

\section{Otros documentos consultados}

Agüeros, V. (14 de diciembre de 1894). [Publicidad comercial]. El Tiempo, p. 2.

Agencia Reforma. (20 de mayo de 2017). Prevé OCDE aumento de obesidad en México.

https://www.elsiglodetorreon.com.mx/noticia/1342532.preveocdeaumentodeobes idml

Muro, M. (28 de marzo de 1904). [Publicidad comercial]. El Contemporáneo, p. 4. 